\title{
Increased expression of hepcidin and associated upregulation of JAK/STAT3 signaling in human gastric cancer
}

\author{
ERDONG ZUO*, YE LU*, MIN YAN, XIANGTAO PAN and XU CHENG \\ Department of Chemotherapy and Hematology, Taicang Affiliated Hospital of Soochow University, \\ Suzhou, Jiangsu 215400, P.R. China
}

Received April 20, 2016; Accepted July 3, 2017

DOI: $10.3892 / \mathrm{ol} .2017 .7574$

\begin{abstract}
Gastric cancer is one of the most common malignancies with high cancer-associated mortality rate globally. Hepcidin is the peptide hormone, which is critically important in the regulation of systemic iron homeostasis. Cumulating evidence has reported that the disturbed local expression of hepcidin may serve as a predictive biomarker in assessing the clinical outcomes in a range of cancer types. However, the expression profile of hepcidin in human gastric cancer is remains to be investigated. In the present retrospective study, using archived paraffin-embedded tissue blocks, the local production of hepcidin by immunohistochemical analysis was detected, and then its correlation with clinicopathological characteristics in human gastric cancer was evaluated. In parallel, using western blotting, quantitative reverse transcription polymerase chain reaction and chromatin immunoprecipitation assay, the local status of Janus kinase (JAK)/signal transducer and activator of transcription 3 (STAT3) signaling in response to inflammatory stimuli mediated by interleukin (IL)-6, which in turn regulates transcriptional activity of hepcidin gene (HAMP) was also assessed. The results indicated that, the local production of hepcidin was significantly elevated in tumor tissues compared with adjacent non-tumor tissues, and was tightly correlated with increasing tumor stages according to the tumor node metastasis (TNM) classification. In addition, JAK/STAT3 signaling and the STAT3 binding affinity to the HAMP gene promoter were significantly enhanced, in parallel with an increased expression of hepcidin, in tumor tissues compared with adjacent non-tumor tissues. Collectively, the present study indicated that local expression of hepcidin in gastric cancer tumor tissues was positively correlated with
\end{abstract}

Correspondence to: Professor $\mathrm{Xu}$ Cheng, Department of Chemotherapy and Hematology, Taicang Affiliated Hospital of Soochow University, 58 Changsheng South Road, Suzhou, Jiangsu 215400, P.R. China

E-mail: chengxu_0512@126.com

*Contributed equally

Key words: gastric cancer, hepcidin, interleukin-6, Janus kinase/signal transducer and activator of transcription 3 signaling increasing tumor stages, which may be closely associated with the upregulation of IL-6-mediated JAK/STAT3 signaling in human gastric cancer.

\section{Introduction}

Gastric cancer is the fourth most common type of cancer and the second leading cause of cancer-associated mortality globally, and its incidence was estimated to be approximately one million per year worldwide (1). According to the multi-factorial and multi-step model in the pathogenesis of gastric cancer, apart from genetic alterations, environmental factors are also markedly involved in different stages of carcinogenesis $(2,3)$. Recently, cumulating evidence reported that high intake of heme iron (e.g., fresh and processed red meat) or low body iron store (e.g., iron-deficiency anemia) was correlated with an increased risk of gastric cancer, indicating that altered iron metabolism may be mediated in the development of gastric cancer (4-7). Furthermore, novel findings indicated that iron-chelating agents, including deferoxamine and deferasirox might potentially exert anti-proliferative effects on gastric cancer cells by inducing apoptosis (8). Accordingly, the altered iron metabolism may have an extensive role in the development of gastric carcinogenesis.

Hepcidin is the peptide hormone, which is primarily synthesized by hepatocytes in the liver and secreted into the circulation to effectively regulate systemic iron homeostasis (9). It is generally believed that, by binding to its target receptor, ferroportin, hepcidin would inhibit iron absorption from duodenal enterocytes and iron release from macrophages and hepatocytes, which is mediated by rapid endocytosis and degradation of the hepcidin-ferroportin complex (10). Disordered hepcidin signaling may lead to several iron-restrictive and iron-overload diseases, including iron deficiency anemia and hereditary hemochromatosis $(11,12)$. Previously, it has been reported that increased circulating levels of hepcidin are associated with a range of malignancies (13-15). Of note, apart from the liver as the major place for hepcidin synthesis, various other organs including cancer tissues may locally synthesize and secret hepcidin (16). Notably, studies have demonstrated that altered expression of hepcidin in tumor tissues may serve as a predictive biomarker in assessing the clinical outcomes of several types of cancer $(13,17)$. Furthermore, it was indicated that aberrant hepcidin signaling might promote tumor growth 
in breast cancer (18). However, in human gastric cancer, there remain to be limited data on the expression profile of hepcidin in tumor tissues and its correlation with the clinicopathological characteristics in gastric cancer.

Previous studies have indicated that hepcidin is a mature defensin-like peptide containing 25 amino acid residues and 4 disulfide bonds, which is cleaved intracellularly from the preprohormone encoded by the gene named hepcidin antimicrobial peptide (HAMP), which is located at the locus $19 q 13$ in the human genome (19). It is widely considered that the production of hepcidin is predominately controlled at the level of transcription, which is rapidly increased by various inflammatory stimuli (20). In particular, in response to pro-inflammatory cytokines, including interleukin (IL)-6, the stimulatory effects on hepcidin expression is largely mediated through the activation of Janus kinase/signal transducer and activator of transcription 3 (JAK/STAT3) signaling. Therefore, to promote the transcriptional activity of hepcidin, this would depend on the interaction between STAT3 and the related STAT3-binding element in the promoter region of the HAMP gene (21-23). Indeed, regardless of underlying etiologies, including diet and Helicobacter pylori infection, the association between chronic inflammation and gastric cancer has been well established (24). Nevertheless, whether and to what extent the inflammation-induced JAK/STAT3 signaling would be involved in the regulation of hepcidin expression in human gastric cancer remains to be investigated.

The aim of the present study was to detect the expression of hepcidin and then to assess its correlation with the clinicopathological characteristics in human gastric cancer. It was further determined whether altered hepcidin expression in human gastric cancer might be associated with the status of the JAK/STAT3 signaling pathway as this may regulate the expression of hepcidin at the transcriptional level. Consequently, the prognostic value of hepcidin for gastric cancer was evaluated. Additionally, the mechanistic underpinnings affecting hepcidin expression at the cellular and molecular level were preliminarily investigated so as to provide a potential target to correct aberrant local expression of hepcidin in gastric cancer.

\section{Materials and methods}

Patients and tissue specimens. A total of 62 gastric cancer patients, who were treated by curative gastrectomy at Taicang Affiliated Hospital of Soochow University (Suzhou, China) from February 2009 to February 2014, were enrolled in the present study. The characteristics of the 62 gastric cancer patients were summarized in Table I. All patients did not receive chemotherapy and/or radiotherapy prior to surgery and did not have a history of concurrent tumors. The 62 archived formalin-fixed paraffin-embedded tumor tissue blocks of the aforementioned patients, which were obtained by biopsy, were collected for the experiments. Additionally, 15 randomly selected tissues adjacent to the tumor confirmed by pathological diagnosis as normal gastric mucosal tissues were selected as the non-tumor group. The tumor grade and clinical stage were determined according to the tumor node metastasis (TNM) classification for gastric cancer (7th Edition of the AJCC Cancer Staging Manual) (25). Anemia was defined according to the following criteria for hemoglobin
Table I. Clinicopathological characteristics of patients with gastric cancer.

\begin{tabular}{lc} 
Characteristics & Number of cases, $\mathrm{n}(\%)$ \\
\hline Age, years & \\
$\leq 60$ & $28(45.2)$ \\
$>60$ & \\
Gender & $40(64.8)$ \\
Male & $22(35.5)$ \\
Female & \\
Anemia & $33(53.2)$ \\
Negative & $29(46.8)$ \\
Positive & \\
T categories & $23(37.1)$ \\
T1 and T2 & $39(62.9)$ \\
T3 and T4 & \\
Lymph node metastasis & $27(43.5)$ \\
Negative & $35(56.5)$ \\
Positive & \\
Other metastasis & $53(85.5)$ \\
Negative & $9(14.5)$ \\
Positive &
\end{tabular}

concentration: $<120.0 \mathrm{~g} / 1$ for males and $<110.0 \mathrm{~g} / \mathrm{l}$ for females. Written informed consent was obtained from all patients and approved by the Committee on Human Rights in Research. The present study was conducted in accordance with the Declaration of Helsinki, and ethical approval was obtained from the Institutional Review Board at the Taicang Affiliated Hospital of Soochow University.

Immunohistochemical analysis. All paraffin-embedded tissues were consecutively cut into 4 sections (thickness, $5 \mu \mathrm{m}$ ). For each sample, one section was used to confirm the pathological diagnosis by hematoxylin-eosin staining (hematoxylin-stained for $\sim 10 \mathrm{~min}$ and eosin-stained for $\sim 2 \mathrm{~min}$ at $30^{\circ} \mathrm{C}$ ). The other three sections were detected by immunohistochemical analysis according to the following procedures. According to the streptavidin-biotin-peroxidase complex immunohistochemical protocol (Thermo Fisher Scientific, Inc., Waltham, MA, USA), the paraffin-embedded sections were deparaffinized with xylene and rehydrated in the decreasing concentrations of ethanol, followed by incubating in $3 \% \mathrm{H}_{2} \mathrm{O}_{2}$ for $30 \mathrm{~min}$ at room temperature. Then, the slides were incubated in $10 \mathrm{mM}$ citrate buffer ( $\mathrm{pH}$ 6.0) for $20 \mathrm{~min}$ for antigen retrieval, and immersed in phosphate-buffered saline (PBS) containing 15\% goat serum (Thermo Fisher Scientific, Inc.) for $30 \mathrm{~min}$ at room temperature. The rabbit anti-human hepcidin polyclonal primary antibody (1:200; catalog no. ab30760; Abcam, Cambridge, MA, USA) was added and incubated overnight at $4^{\circ} \mathrm{C}$. Following washing with PBS, goat anti-rabbit horseradish peroxidase-labeled secondary antibody (1:200; catalog no. BA1088; Wuhan Boster Biological Technology, Ltd., Wuhan, China) was added and incubated for $30 \mathrm{~min}$ at room temperature. By rinsing with PBS, the slides 

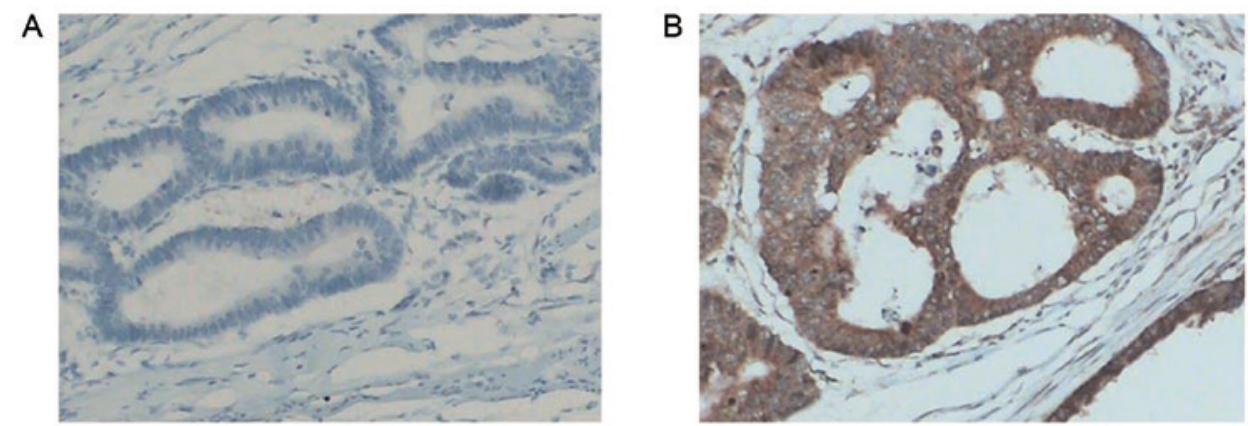

Figure 1. Immunohistochemical staining of hepcidin in the human gastric mucosal epithelium (magnification, x100). (A) Negative immunoreactivity for hepcidin. (B) Positive immunoreactivity for hepcidin.

were then counterstained using hematoxylin for $10 \mathrm{~min}$ at $30^{\circ} \mathrm{C}$. Following dehydrating in increasing concentrations of ethanol, the slides were mounted, and cover slips were placed for the next microscopic evaluation.

Immunohistochemical staining was scored independently by three researchers who were blinded to the clinicopathological data. Microscopic evaluation was performed under five random visual fields at a magnification of x200 (Leica Microsystems GmbH, Wetzlar, Germany). Staining results for hepcidin were classified by estimating the percentage of epithelial cells exhibiting specific immunoreactivity (Fig. 1). Immunoreactivity was scored as follows: Negative, no immunoreactivity; weak, $<33 \%$ positive cells; moderate, 33-67\% positive cells; and strong, $>67 \%$ positive cells. Samples that exhibited negative and weak immunoreactivity were considered as negative, and those exhibited moderate and strong immunoreactivity were considered as positive.

Western blot analysis. The protein samples were routinely extracted from formalin-fixed paraffin-embedded tissue blocks using liquid tissue buffer (Expression Pathology Inc., Rockville, MD, USA) for homogenizing in dry ice, then incubated at $95^{\circ} \mathrm{C}$ for $90 \mathrm{~min}$. Using the protein extraction NP-40 lysis buffer (Thermo Fisher Scientific Inc.) at $100^{\circ} \mathrm{C}$ for $20 \mathrm{~min}$ followed by a $2 \mathrm{~h}$ incubation at $80^{\circ} \mathrm{C}$, the protein concentration was determined using the Bradford method (Quick Start ${ }^{\mathrm{TM}}$ Bradford Protein Assay; Bio-Rad Laboratories, Inc., Hercules, CA,USA). Samples with equal quantities of protein $(80 \mu \mathrm{g})$ were then loaded in each lane for electrophoresis using 0.1\% SDS, $10 \%$ polyacrylamide gel and $4 \%$ polyacrylamide stacking gel. Proteins were subsequently transferred to polyvinyl difluoride membranes (EMD, Billerica, MA, USA). Each membrane was treated with Tris-buffered PBS containing 5\% bovine serum albumin (BSA) (Sigma-Aldrich; Merck KGaA, Darmstadt, Germany) and 0.1\% v/v Tween-20 (Sigma-Aldrich; Merck $\mathrm{KGaA}$ ) with gentle shaking for $1 \mathrm{~h}$ at room temperature. This was followed by incubation overnight at $4^{\circ} \mathrm{C}$ with primary antibodies against interleukin 6 signal transducer (gp130; 1:1,000; catalog no. sc-9045; Santa Cruz Biotechnology, Inc., Dallas, TX, USA), JAK1 (1:1,000; catalog no. sc-7228; Santa Cruz Biotechnology, Inc.) and STAT3 (1:1,000; catalog no. sc-482; Santa Cruz Biotechnology, Inc.), respectively. The membranes were washed for $10 \mathrm{~min}$ for three times in TBST solution and further incubated at room temperature for $10 \mathrm{~min}$ with the secondary horseradish peroxidase-conjugated goat anti-rabbit antibody $(1: 5,000)$. Proteins were then visualized using ECL reagent (Amersham; GE Healthcare Bio-Sciences, Pittsburgh, PA, USA) and then exposed to X-ray film. The $\beta$-actin protein (1:1,000; catalog no. sc-47778; Santa Cruz Biotechnology, Inc.) was used as the internal control for normalizing the relative density. Results were quantified and analyzed (three repeats performed for each sample) with Kodak electrophoresis documentation and analysis system, and Kodak ID image analysis software (Kodak, Rochester, NY, USA).

Reverse transcription-quantitative polymerase chain reaction $(R T-q P C R)$. The total tissue RNA was routinely isolated from formalin-fixed paraffin-embedded tissue blocks. Reverse transcription and first strand cDNA synthesis was performed using MMLV-RT reverse transcriptase (three repeats performed for each sample) (Invitrogen; Thermo Fisher Scientific, Inc.). RT-qPCR analysis was employed to analyze the gene expression of HAMP, IL-6, gp130, JAK1 and STAT3. The GAPDH gene was used as an internal reference, and serial dilutions of the positive control were performed on each plate to create a standard curve. The primer sequences for the genes are as follows: HAMP forward, 5'-TCTGCTTTCACAGACGGG AC-3', and reverse 5'-CTTAGCACAGACACTCGGCA-3'; IL-6 forward, 5'-AACCTGAACCTTCCAAAGATGG-3', and reverse 5'-TCTGGCTTGTTCCTCACTACT-3'; gp130 forward, 5'-TGAAGCCATAGTCGTGCCTG-3', and reverse 5'-ACTGGACAGTGCTCGAAGTG-3'; JAK1 forward 5'-TCT ATGAAAGCCGGTGCAGG-3', and reverse 5'-CCTGTATTG TCTTCGGGGTCA-3'; STAT3 forward 5'-GCCCTTTGG AACGAAGGGTA-3', and reverse 5'-ATGGTATTGCTGCAG GTCGT-3'; GAPDH forward 5'-GCATCTTCTTTTGCGTCG CC-3', and reverse 5'-AGTGATGGCATGGACTGTGG-3'. RT-qPCR was performed with a $25 \mu 1$ reaction mixture in a 96-well plate (Takara, Japan) and a thermocycler (iCycler iQ; Bio-Rad Laboratories, Inc.). The expression of target gene was normalized to the reference GAPDH to obtain the relative threshold cycle $(\Delta \mathrm{Cq})$ and $2^{-\Delta \Delta \mathrm{Cq}}$ was subsequently used to determine the relative abundance of target gene expression between each group.

Chromatin immunoprecipitation assay (ChIP). With the use of commercially available kit (CHIP-IT ${ }^{\circledR}$ FFPE; Active Motif, Carlsbad, CA, USA), ChIP was performed on formalin-fixed paraffin-embedded gastric cancer tissues, according to the manufacturer's instructions. Specifically, the sonicated 
Table II. Correlation between local expression of hepcidin and clinicopathological characteristics.

\begin{tabular}{|c|c|c|c|c|c|c|}
\hline \multirow[b]{2}{*}{ Characteristics } & \multicolumn{2}{|c|}{$\begin{array}{l}\text { Local expression of } \\
\text { hepcidin in tumor tissues }\end{array}$} & \multirow[b]{2}{*}{$\chi^{2}$} & \multirow[b]{2}{*}{ P-value } & \multirow[b]{2}{*}{$\begin{array}{c}\text { Spearman's } \\
\text { correlation coefficient }\end{array}$} & \multirow[b]{2}{*}{ P-value } \\
\hline & $\begin{array}{c}\text { Number of } \\
\text { negative cases }\end{array}$ & $\begin{array}{c}\text { Number of } \\
\text { positive cases }\end{array}$ & & & & \\
\hline Age, years & & & 0.172 & 0.678 & 0.053 & 0.684 \\
\hline$\leq 60$ & 13 & 15 & & & & \\
\hline$>60$ & 14 & 20 & & & & \\
\hline Gender & & & 0.051 & 0.822 & -0.029 & 0.826 \\
\hline Male & 17 & 23 & & & & \\
\hline Female & 10 & 12 & & & & \\
\hline T stages & & & 6.984 & $0.008^{\mathrm{a}}$ & 0.336 & $0.008^{\mathrm{a}}$ \\
\hline $\mathrm{T} 1$ and $\mathrm{T} 2$ & 15 & 8 & & & & \\
\hline $\mathrm{T} 3$ and $\mathrm{T} 4$ & 12 & 27 & & & & \\
\hline Lymph node metastasis & & & 0.412 & 0.521 & 0.081 & 0.529 \\
\hline Negative & 13 & 14 & & & & \\
\hline Positive & 14 & 21 & & & & \\
\hline Other metastasis & & & 0.447 & 0.503 & 0.085 & 0.512 \\
\hline Negative & 24 & 29 & & & & \\
\hline Positive & 3 & 6 & & & & \\
\hline Anemia & & & 1.821 & 0.177 & 0.171 & 0.183 \\
\hline Negative & 17 & 16 & & & & \\
\hline Positive & 10 & 19 & & & & \\
\hline
\end{tabular}

${ }^{\mathrm{a}} \mathrm{P}<0.01$.

chromatin was used for the immunoprecipitation reaction. Sonicated chromatin ( $\geq 200 \mathrm{ng}$ per reaction), ChIP buffer and protease inhibitor cocktail were added in order in the $1.5 \mathrm{ml}$ microcentrifuge tube. The rabbit monoclonal anti-STAT3 (1:100; catalog no. ab68153; Abcam, Cambridge, UK) was transferred and incubated overnight at $4^{\circ} \mathrm{C}$. Protein $\mathrm{G}$ agarose beads were subsequently added and incubated at room temperature for $3 \mathrm{~h}$ following extensive blocking in $0.5 \%$ BSA for $2 \mathrm{~h}$ at room temperature. Subsequently, following washing and reversing the cross-links, DNA was recovered and purified. Finally, the commercially available CYBR Green (Bio-Rad Laboratories, Inc.) quantitative PCR was performed with primers (forward, 5'-GAGGGTGACACAACCCTGTT-3', and reverse, 5'-CGAGTGACAGTCGCTTTT-3') flanking the 155 bp region containing the putative STAT3 binding site in the promoter region of the human HAMP gene. The data was expressed as the percent of input.

Statistical analysis. For the categorical variables, differences between groups were calculated using $\chi^{2}$ test or Fisher's exact test. Bivariate correlations between variables were examined by Spearman's correlation analysis. For the continuous variables, all data are expressed as the mean \pm standard deviation. One-way analysis of variance with Tukey's post hoc test was used to evaluate the differences between certain groups in western blotting experiments. With regards to the differences of mRNA levels between each group, $\mathrm{P}<0.05$ was considered to indicate a statistically significant difference when the ratio of $2^{-\Delta \Delta \mathrm{Cq}}>1.7$. Statistical significance was accepted at a level of $\mathrm{P}<0.05$. SPSS statistical software (version 18.0; SPSS, Inc., Chicago, IL, USA) was used for these statistical analyses.

\section{Results}

Local expression of hepcidin in human gastric cancer. Using immunohistochemical analysis, the local production of hepcidin was extensively evaluated in tumor and adjacent non-tumor tissues of gastric cancer. The local positive expression rate of hepcidin in tumor tissues $(35 / 62,56.5 \%)$ was significantly higher compared with adjacent non-tumor tissues $(3 / 15,20.0 \%)(\mathrm{P}<0.05$; data not shown). With respect to the $\mathrm{T}$ categories in tumor tissues, the local positive expression rate of hepcidin in T3 and T4 stages $(27 / 39,69.2 \%)$ was significantly elevated compared with $\mathrm{T} 1$ and $\mathrm{T} 2$ stages $(8 / 23,34.8 \%)(\mathrm{P}<0.05$; Table II). In addition, with respect to lymph node metastasis, there was no significant difference between negative (14/27, $51.9 \%)$ and positive $(21 / 35,60.0 \%)$ groups. Furthermore, with regards to metastasis (other than lymph node metastasis), there was no significant difference between negative $(29 / 53,54.7 \%)$ and positive $(6 / 9,66.7 \%)$ groups (Table II).

HAMP mRNA expression was extensively evaluated in tumor and adjacent non-tumor gastric cancer tissues using RT-qPCR. The mRNA expression of HAMP in tumor tissues was significantly higher compared with adjacent non-tumor tissues $(\mathrm{P}<0.05$; Fig. $2 \mathrm{~A})$. With respect to the $\mathrm{T}$ stages, the mRNA expression of HAMP in tumor tissues at T3/T4 
A

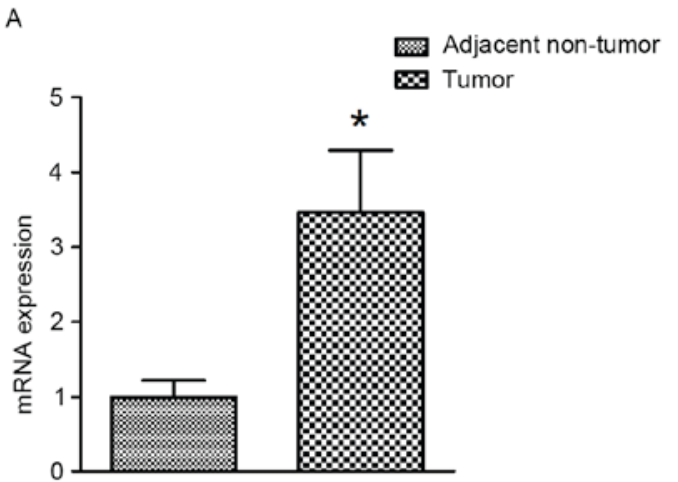

C

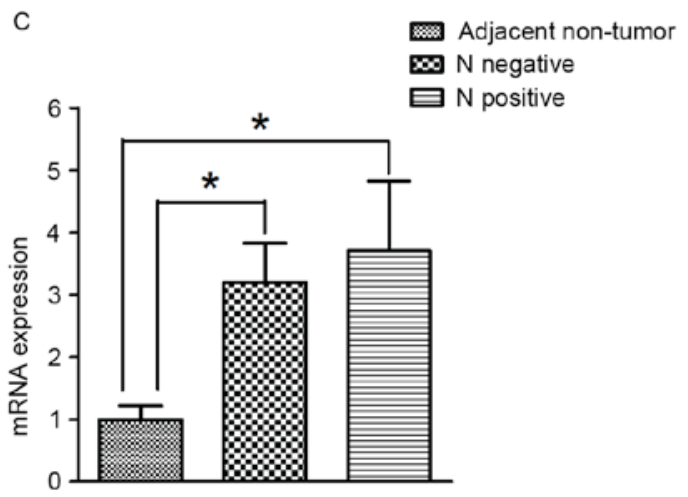

B
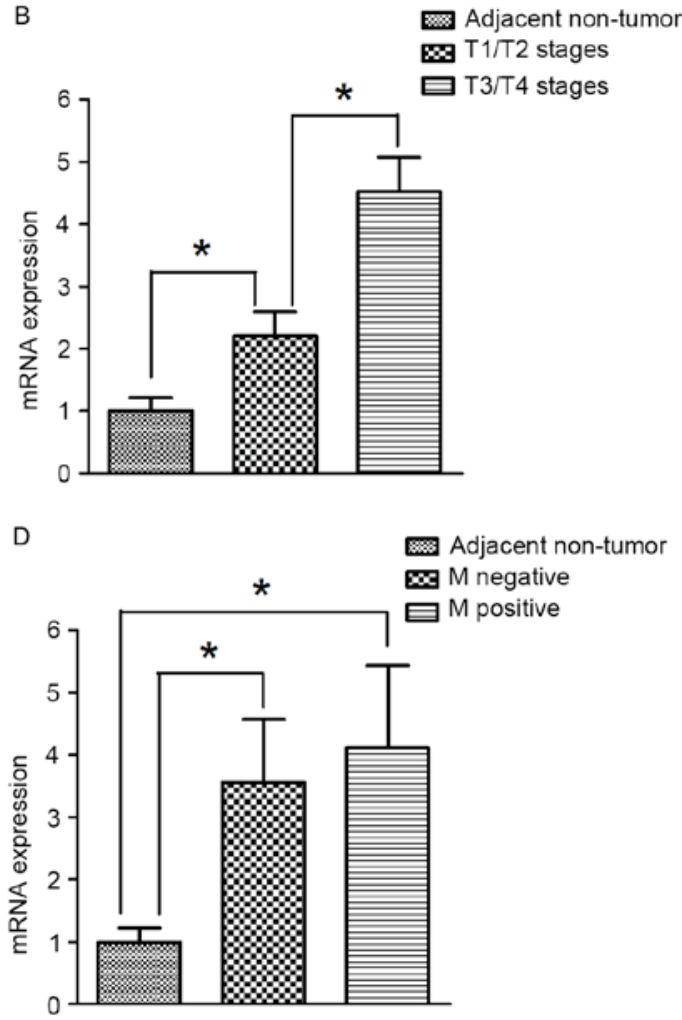

Figure 2. mRNA expression of HAMP in human gastric cancer tissues. (A) The mRNA expression of HAMP in tumor tissues (n=62) was significantly higher compared with adjacent non-tumor tissues $(n=15)$. (B) The mRNA expression of HAMP in tumor tissues from patients at stages T3/T4 ( $n=39$ ) was significantly increased compared with tissues from patients at stages T1/T2 $(n=23)$, which were both significantly higher compared with the expression in adjacent non-tumor tissues $(n=15)$. (C) The mRNA expression of HAMP in tumor tissues of negative ( $\mathrm{N}$ negative group, $\mathrm{n}=27)$ and positive lymph node metastasis ( $\mathrm{N}$ positive group, $\mathrm{n}=35$ ) were significantly higher compared with the expression in adjacent non-tumor tissues $(\mathrm{n}=15)$. However, there were no significant differences in HAMP mRNA expression between negative and positive lymph node metastasis groups. (D) The mRNA expression of HAMP in tumor tissues of negative (M negative group, $\mathrm{n}=53$ ) and positive metastasis (with the exception of lymph node metastasis) $(\mathrm{n}=9$ ) were significantly higher compared with adjacent non-tumor tissues $(\mathrm{n}=15)$. However, there were no significant differences in HAMP mRNA expression between groups of negative and positive metastasis (with the exception of lymph node metastasis). Values are presented as the mean \pm standard deviation. ${ }^{*} \mathrm{P}<0.05$. HAMP, hepcidin antimicrobial peptide.

stages was significantly elevated compared with T1/T2 stages $(\mathrm{P}<0.05$; Fig. 2B). Additionally, there were no significant differences in HAMP expression between positive and negative lymph node metastasis groups (Fig. 2C). Similarly, with regards to metastasis (excluding lymph node metastasis), there were also no significant differences between in HAMP expression between negative and positive groups (Fig. 2D).

Expression of gp130, JAK1, and STAT3 proteins in human gastric cancer tissues. In order to compare the differences in JAK/STAT3 signaling in response to inflammatory stimuli mediated by IL- 6 in human gastric cancer, protein expression of the associated ligand-receptor and intracellular regulators were detected in the tumor and adjacent non-tumor tissues. Western blot analyses indicated that the expression of gp130, JAK1, and STAT3 proteins were significantly higher in gastric cancer tumor tissues compared with adjacent non-tumor tissues (Fig. 3A-C). In addition, the local protein expression of JAK1 and STAT3 in T3/T4 stages was significantly elevated in gastric cancer tumor tissues compared with T1/T2 stages (Fig. 3B and C). However, there were no significant differences in gp130 expression between T1/T2 and T3/T4 (Fig. 3A).

mRNA expression of IL-6, gp130, JAK1 and STAT3 in human gastric cancer tissues. Similar to the results for protein expression, RT-qPCR results indicated that the mRNA expression of IL-6, gp130, JAK1, and STAT3 was significantly higher in tumor tissues compared with adjacent non-tumor tissues (Fig. 4A-D). In addition, the expression of JAK1 and STAT3 in T3/T4 stage gastric cancer tumor tissues was significantly increased compared with expression in T1/T2 gastric cancer tumor tissues (Fig. 4C and D). However, in tumor tissues, there were no significant differences in IL-6 and gp130 expression between T3/T4 and T1/T2 stages (Fig. 4A and B).

Binding capacity of STAT3 to the HAMP gene promoter of in human gastric cancer. To further evaluate the function of STAT3 as a trans-acting regulator that stimulate the transcriptional activity of the HAMP gene, the binding affinity of STAT3 on the concerned element in the promoter region of the HAMP gene was determined by ChIP assay in paraffin-embedded gastric cancer tissues. ChIP analyses indicated that the binding affinity of STAT3 to the promoter region of HAMP gene was significantly higher in tumor tissues compared with adjacent non-tumor tissues in human gastric cancer. In addition, the binding affinity of STAT3 to the promoter region in the HAMP gene was significantly more elevated in tumor tissues at $\mathrm{T} 1 / \mathrm{T} 2$ stages compared with T3/T4 (Fig. 5). 

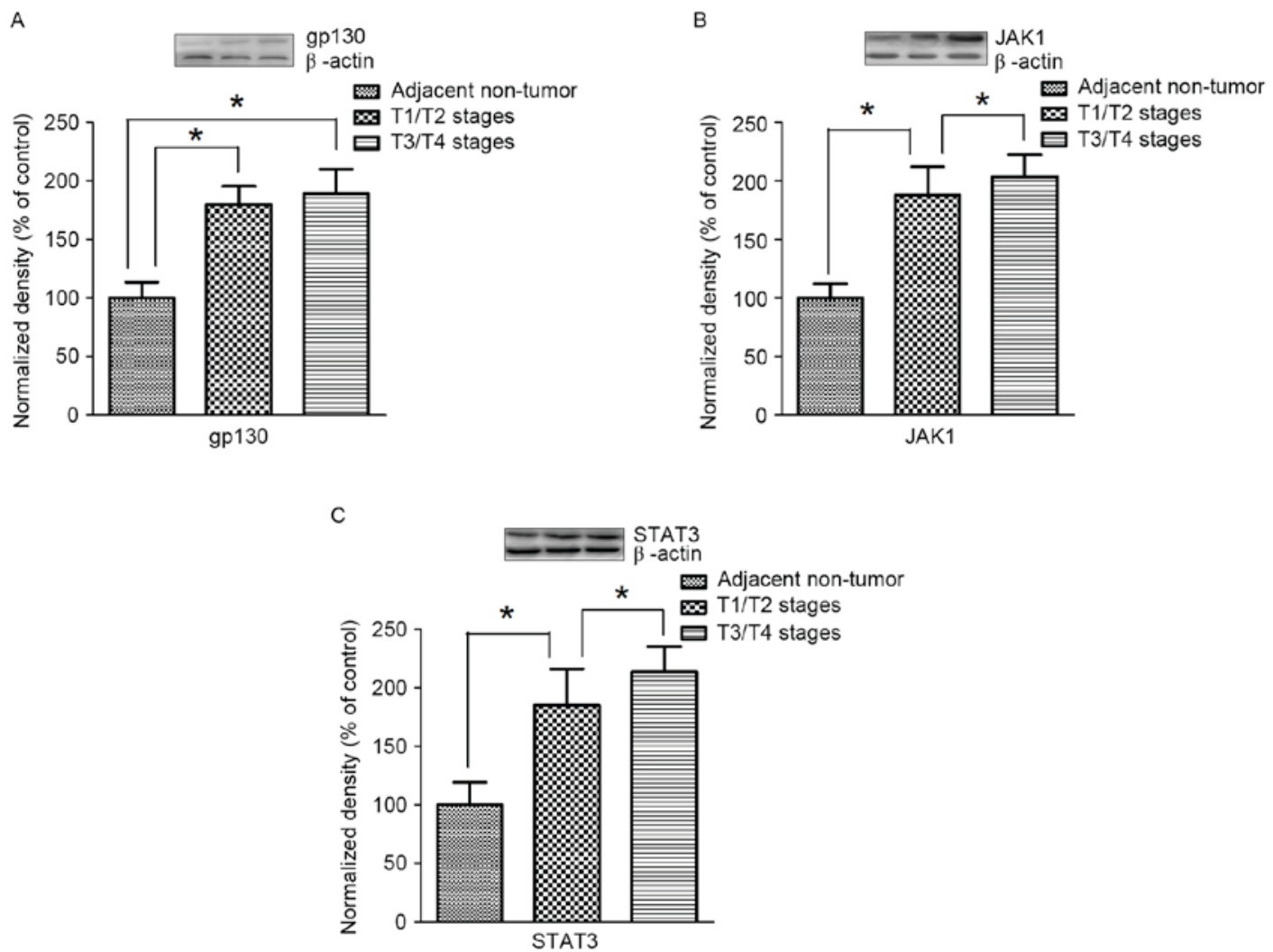

Figure 3. Protein expression of gp130, JAK1 and STAT3 in human gastric cancer tissues. (A) The protein expression of gp130 in tumor tissues of different T stages were significantly higher compared with the expression in adjacent non-tumor tissues $(n=15)$. However, there were no significant differences in gp130 protein expression between T1/T2 ( $\mathrm{n}=23$ ) and T3/T4 $(\mathrm{n}=39)$. (B) The protein expression of JAK1 in tumor tissues of T3/T4 stages ( $\mathrm{n}=39)$ was significantly increased compared with T1/T2 stages $(n=23)$, which were both significantly higher compared with the expression in adjacent non-tumor tissues $(n=15)$. (C) The protein expression of STAT3 in tumor tissue of T3/T4 stages $(n=39)$ was significantly elevated compared with T1/T2 stages $(n=23)$, which were both significantly higher compared with adjacent non-tumor tissues $(n=15)$. Values are expressed as the mean \pm standard deviation. " $\mathrm{P}<0.05$. gp 130 , interleukin 6 signal transducer; JAK1, Janus kinase 1; STAT3, signal transducer and activator of transcription 3.

\section{Discussion}

Due to its capacity to generate deleterious free radicals, the disturbed iron metabolism not only has the ability to damage pivotal macromolecules, including DNA, but also mediate diverse pathogenic signaling, such as hypoxia-inducible factor and Wnt signaling pathways, which are toxic and carcinogenic (26-29). Accruing epidemiological evidence has revealed that altered hepcidin signaling is tightly associated with the clinical outcomes in cancer patients and may be a prognostic biomarker in malignancy, including breast cancer $(30,31)$. In fact, a previous study by the present authors demonstrated that the local production of hepcidin in breast cancer tissue was prominently increased (15). Moreover, the expression of hepcidin may be essentially regulated by inflammation in response to pro-inflammatory cytokines, including IL-6 (10). Indeed, chronic inflammation has critical roles in the carcinogenesis of gastric cancer (24). As such, the present authors hypothesize that local hepcidin production may be altered in the development of human gastric cancer and be associated with the altered inflammatory responses mediated by IL- 6 . In the present retrospective study on formalin-fixed paraffin-embedded patient samples, it was demonstrated that the local production of hepcidin was significantly elevated positively correlated with increasing tumor stages. In addition, the local JAK/STAT3 signaling associated with IL-6 was significantly increased in parallel with the expression of hepcidin, which was able to stimulate the transcriptional activity of the hepcidin gene.

Previous studies have demonstrated that the intracellular iron regulation is modified in a range of malignancies (28). In particular, elevating intracellular iron levels may enhance Wnt signaling, which is closely associated with increased cellular proliferation in the pathogenesis of colorectal cancer (26). In general, the disorder of iron homeostasis in cancer may occur through changes in iron flow (uptake and efflux) and storage, both of which are controlled by dozens of iron-regulatory proteins (28). Hepcidin, which can be locally produced in the tumor, is the master protein for regulating cellular iron flow (32). Together with the iron efflux pump in vertebrates, ferroportin, the local hepcidin-ferroportin axis has a key role in the regulation of autocrine and/or paracrine iron regulatory loop in cancer (33). When intracellular iron storage and systemic iron levels are elevated, hepcidin expression is induced to bind with ferroportin and trigger its subsequent lysosomal degradation $(10,34)$. In cancer cells, the local expression of hepcidin is elevated, together with the low levels of ferroportin, to synergistically decrease the iron efflux to generate the unstable iron pool to satisfy the increased metabolic needs in cancer $(33,35)$. Furthermore, a previous study indicated that the increased expression of hepcidin in colorectal cancer tissues was correlated with 
A

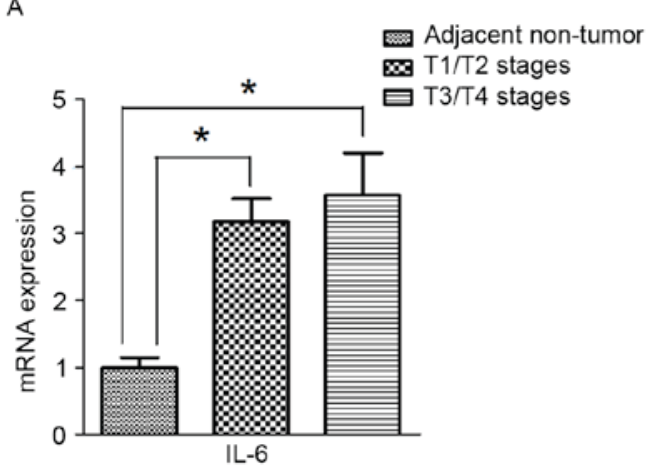

C

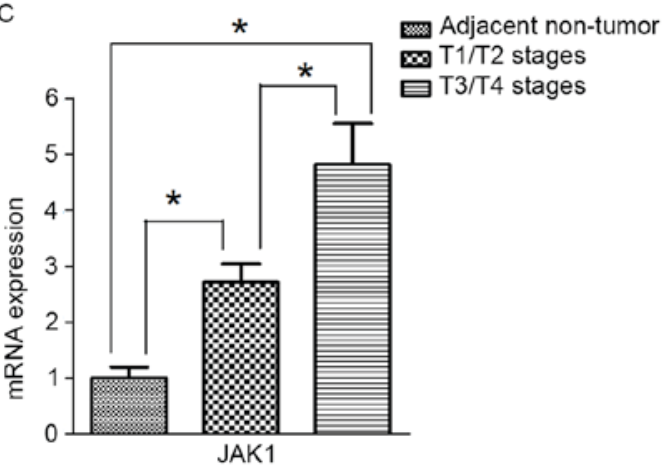

B
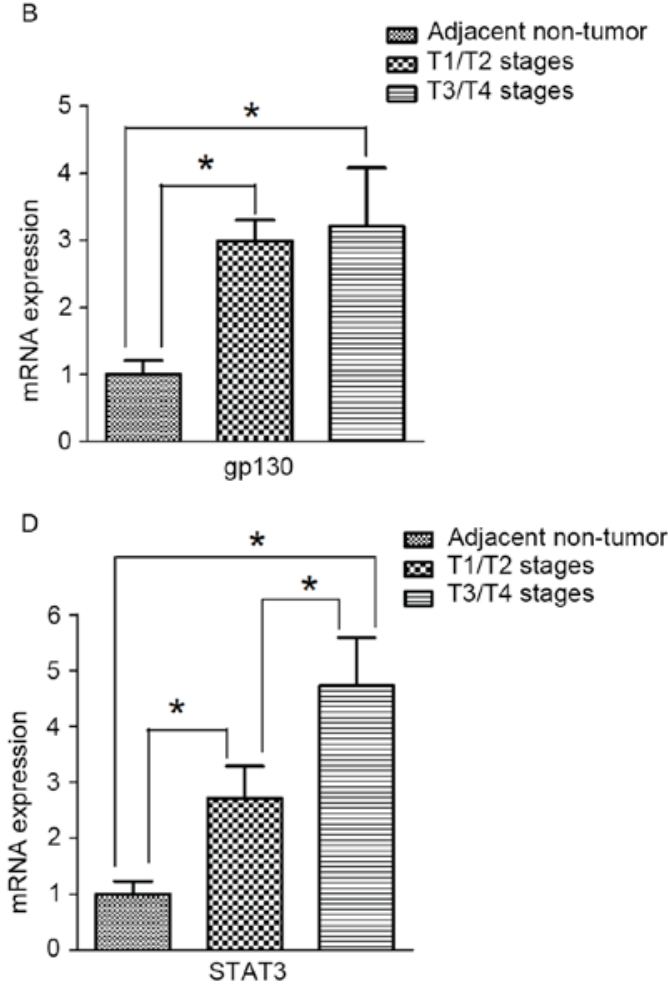

Figure 4. mRNA expression of IL-6, gp130, JAK1 and STAT3 in human gastric cancer tissues. (A) The mRNA expression of IL-6 in tumor tissues from patients at different $T$ stages were significantly higher compared with adjacent non-tumor tissues $(n=15)$. However, there were no significant differences in IL-6 expression between T1/T2 $(n=23)$ and T3/T4 $(n=39)$. (B) The mRNA expression of gp130 in tumor tissues of different $\mathrm{T}$ stages were significantly higher compared with adjacent non-tumor tissues $(n=15)$. However, there were no significant differences in gp130 expression between T1/T2 ( $n=23)$ and T3/T4 $(n=39)$. (C) The mRNA expression of JAK1 in tissues from patients with tumors at T3/T4 stages $(n=39)$ was significantly increased compared with the expression in tumor tissues at T1/T2 stages $(n=23)$, which were both significantly higher compared with adjacent non-tumor tissues ( $\mathrm{n}=15)$. (D) The mRNA expression of STAT3 in tumor tissues at T3/T4 stages $(\mathrm{n}=39)$ was significantly increased compared with the expression in tumor tissues at $\mathrm{T} 1 / \mathrm{T} 2$ stages ( $\mathrm{n}=23$ ), which were both significantly higher compared with adjacent non-tumor tissues $(n=15)$. Values are expressed as the mean \pm standard deviation. ${ }^{*} \mathrm{P}<0.05$. gp130, interleukin 6 signal transducer; IL-6, interleukin-6; JAK1, Janus kinase 1; STAT3, signal transducer and activator of transcription 3.

increasing T-stage, according to TNM classification (36). In the present study, results from immunohistochemistry and RT-qPCR showed that local hepcidin production and mRNA expression of HAMP were significantly increased in gastric cancer tumor tissues compared with adjacent non-tumor tissues. In addition, the local hepcidin production and mRNA expression of HAMP in tumor tissues at T3/T4 stages was significantly more elevated compared with T1/T2 stages. However, with respect to the $\mathrm{N}$ and $\mathrm{M}$ categories in the TNM classification system, there were no significant differences in local hepcidin production and HAMP mRNA expression between different $\mathrm{N}$ and $\mathrm{M}$ stages. Therefore, the local expression of hepcidin was prominently elevated in tumor tissues and positively correlated with invasive but not metastatic properties of human gastric cancer. Of note, a previous study indicated that increased hepcidin expression in tumor tissues was associated with increased metastatic potential and shorter overall survival in renal cell carcinoma (17). Due to the lack of follow-up data, survival was not analyzed in the present study. Therefore, the prognostic value of local expression of hepcidin in human gastric cancer remains to be further evaluated.

It is generally thought that hepcidin production was mainly regulated at the transcriptional level (19).

In general, the transcriptional activity of HAMP gene may be extensively regulated by various stimuli, including substrate

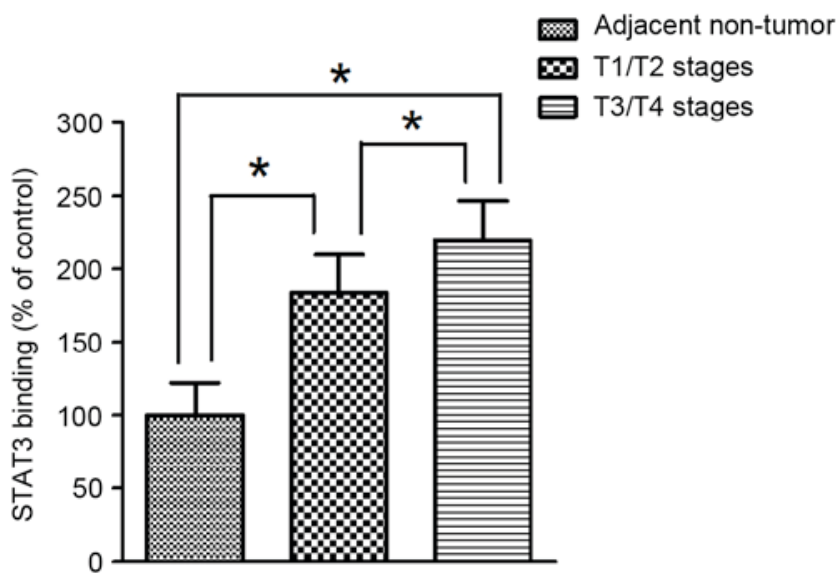

Figure 5. Binding of STAT3 to promoter region in the HAMP gene in human gastric cancer. The binding affinity of STAT3 to the promoter region of HAMP gene in tumor tissue at T3/T4 stages $(n=39)$ was significantly increased compared with $\mathrm{T} 1 / \mathrm{T} 2$ stages $(\mathrm{n}=23$ ), which were both significantly increased compared with adjacent non-tumor tissues $(n=15)$. Values are expressed as the mean \pm standard deviation. ${ }^{*} \mathrm{P}<0.05$. HAMP, hepcidin antimicrobial peptide; STAT3, signal transducer and activator of transcription 3.

(e.g., iron), erythropoietic signals (e.g., erythropoietin) and inflammatory stimuli (37). In particular, in response to inflammation, the pro-inflammatory cytokines, such as IL-6 would 
instigate the downstream signaling to positively upregulate the expression of hepcidin (20). Previous studies indicated that, IL-6 is able to bind to the gp130 protein receptor complex (IL-6 receptor), stimulate JAK tyrosine kinas-mediated phosphorylation of the transcription factor STAT3 $(22,38)$. Subsequently, the activated STAT3 would translocate into the nucleus followed by binding to the STAT3-responsive element on the proximal promoter $(\sim 0.6 \mathrm{~kb}$ fragment of 5'upstream flanking sequence) of the HAMP gene, so as to enhance the transcriptional activity of HAMP (23). In the present study, it was demonstrated that IL-6 mRNA expression was significantly elevated, and both mRNA and protein expressions gp130 were significantly increased in gastric cancer tumor tissues compared with adjacent non-tumor tissues. Additionally, mRNA and protein expression of JAK1 and STAT3 were significantly increased in tumor tissues compared with adjacent non-tumor tissue in human gastric cancer. This indicated that JAK/STAT3 signaling in response to inflammation mediated by IL-6 was prominently enhanced in gastric cancer tumor tissues and may be involved in the pathogenesis of human gastric cancer. In fact, a previous in vitro study in a range of gastric cancer cell lines showed that the broadly expressed IL- 6 and gp130 were able to promote proliferation, invasion and lymphangiogenesis via the JAK/STAT3 signaling pathway (39). Furthermore, the present study showed that, the local mRNA and protein expression of JAK1 and STAT3 in gastric cancer tumor tissues at T3 and T4 stages were significantly increased compared with tumor tissues at T1 and T2 stages. In the light of these findings, the present authors hypothesize that the local elevated expression of hepcidin gastric cancer tissues in increasing $\mathrm{T}$ stages was closely associated with the upregulation of IL-6-mediated JAK/STAT3 signaling pathway.

In order to further assess the trans-acting effects of STAT3 on regulating the transcriptional activity of the HAMP gene, ChIP assay was performed on paraffin-embedded tissue blocks to identify the interactions of STAT3 with specific promoter loci in the HAMP gene in the intact chromatin. The results showed that the binding affinity of STAT3 was significantly increased in gastric cancer tumor tissues compared with adjacent non-tumor tissues. In addition, the binding affinity of STAT3 in tumor tissues at T3/T4 stages was significantly elevated compared with T1/T2 stages. As a consequence, the causal effects of enhanced JAK/STAT3 signaling on the elevated expression of hepcidin in increasing $\mathrm{T}$ stages of human gastric cancer were tentatively verified in the present study. Furthermore, it was previously reported that in human hepatocellular carcinoma, the HAMP gene was transcriptionally repressed in tumor tissues, which was closely associated with the hypermethylated signature in the promoter region (40). However, whether the epigenetic regulation through DNA methylation in the promoter region in the HAMP gene may be altered in human gastric cancer was not determined in the present study and should be further elucidated. Moreover, whether and to what extent the binding affinity of STAT3 would be affected by altered DNA methylation in the promoter region of HAMP gene should be further investigated.

Unlike JAK/STAT3 signaling regulators, there were no significant differences in expression of IL-6 mRNA, gp130 mRNA and protein in gastric cancer tumor tissues between different $\mathrm{T}$ stages. This indicated that upregulation of JAK/STAT3 signaling in increasing $\mathrm{T}$ stages may not be directly affected by the local IL-6 generated de novo in tumor tissues. In fact, previous studies reported that high levels of pro-inflammatory cytokines, such as IL-6 in serum were correlated with poor prognosis in human gastric cancer (41). As such, it was hypothesized that the systemic level of IL-6 in the T3 and T4 stages may be further increased compared with T1 and T2 stages, which may lead to more upregulation of JAK/STAT3 signaling in the increasing $\mathrm{T}$ stages of human gastric cancer. However, this hypothesis was not verified in the present study and should be further evaluated in future research.

Increasing evidence indicated that the incidence of anemia in cancer patients including gastric cancer was frequent and is tightly associated with the poor clinical outcomes $(42,43)$. Multifactorial pathogenesis is involved in the anemia of cancer patients, of which disturbed iron homeostasis, probably due to the inflammatory stimulus induced by the tumor, has a key role $(44,45)$. Moreover, novel studies indicated that hemoglobin concentration is inversely correlated with the levels of hepcidin in cancer of the upper gastrointestinal tract (46). In the present study, the association of anemia in gastric cancer with the local expression of hepcidin in tumor tissues was assessed. In order to avoid the effects of chemotherapy/radiotherapy and surgery on anemia, the hemoglobin concentrations were detected in gastric cancer patients not treated by chemotherapy/radiotherapy prior to surgery. Results from immunohistochemical analysis and RT-qPCR indicated that there were no significant differences in local hepcidin production and HAMP mRNA expression between anemic and non-anemic gastric cancer patients. Therefore, in contrast to systemic hepcidin levels, the local expression of hepcidin in tumor tissue may not serve as a predictive biomarker for assessing anemia in human gastric cancer.

In conclusion, the findings of the present study showed that elevated local expression of hepcidin in tumor tissues was closely correlated with increasing tumor stages in the development of human gastric cancer, which provides a novel insight into the potential prognostic value of tumor hepcidin expression in clinical practice. Additionally, in the pathogenesis of human gastric cancer, the increased tumor hepcidin expression was tightly associated with the upregulation of the JAK/STAT3 signaling pathway, which may be mediated by IL-6. As such, apart from iron-chelating agents as the therapeutic candidates, drugs targeting IL-6-mediated JAK/STAT3 signaling may also be a potential strategy for correcting disturbed local iron homeostasis in gastric cancer.

\section{Acknowledgements}

The present study was supported by research grants from the Suzhou Science and Technology Development project (grant no. SYSD2011035) and the Jiangsu Science and Research project (grant no. YG201404).

\section{References}

1. Ferlay J, Shin HR, Bray F, Forman D, Mathers C and Parkin DM: Estimates of worldwide burden of cancer in 2008: GLOBOCAN 2008. Int J Cancer 127: 2893-2917, 2010.

2. Correa P: Gastric cancer: Overview. Gastroenterol Clin North Am 42: 211-217, 2013. 
3. González CA, Sala N and Rokkas T: Gastric cancer: Epidemiologic aspects. Helicobacter 18 (Suppl 1): S34-S38, 2013.

4. Epplein M,Zheng W,Li H, Peek RM Jr, Correa P, Gao J, Michel A, Pawlita M, Cai Q, Xiang YB, Shu XO: Diet, Helicobacter pylori strain-specific infection, and gastric cancer risk among Chinese men. Nutr Cancer 66: 550-557, 2014.

5. Fonseca-Nunes A, Agudo A, Aranda N, Arija V, Cross AJ, Molina E, Sanchez MJ, Bueno-de-Mesquita HB, Siersema P, Weiderpass E, et al: Body iron status and gastric cancer risk in the EURGAST study. Int J Cancer 137: 2904-2914, 2015.

6. Jakszyn P, Agudo A, Lujan-Barroso L, Bueno-de-Mesquita HB, Jenab M, Navarro C, Palli D, Boeing H, Manjer J, Numans ME, et al: Dietary intake of heme iron and risk of gastric cancer in the European prospective investigation into cancer and nutrition study. Int J Cancer 130: 2654-2663, 2012.

7. Noto JM and Peek RM Jr: Micronutrients: A double-edged sword in microbial-induced gastric carcinogenesis. Trends Cancer 1: 136-144, 2015

8. Kim JL, Lee DH, Na YJ, Kim BR, Jeong YA, Lee SI, Kang S, Joung SY, Lee SY, Oh SC and Min BW: Iron chelator-induced apoptosis via the ER stress pathway in gastric cancer cells. Tumour Biol 37: 9709-9719, 2016.

9. Park $\mathrm{CH}$, Valore EV, Waring AJ and Ganz T: Hepcidin, a urinary antimicrobial peptide synthesized in the liver. J Biol Chem 276 7806-7810, 2001.

10. Ganz T: Systemic iron homeostasis. Physiol Rev 93: 1721-1741, 2013.

11. Babitt JL and Lin HY: The molecular pathogenesis of hereditary hemochromatosis. Semin Liver Dis 31: 280-292, 2011

12. Zhang DL, Senecal T, Ghosh MC, Ollivierre-Wilson H, Tu T and Rouault TA: Hepcidin regulates ferroportin expression and intracellular iron homeostasis of erythroblasts. Blood 118: 2868-2877, 2011.

13. Chen Q, Wang L, Ma Y, Wu X, Jin L and Yu F: Increased hepcidin expression in non-small cell lung cancer tissue and serum is associated with clinical stage. Thorac Cancer 5: 14-24, 2014.

14. Eisfeld AK, Westerman M, Krahl R, Leiblein S, Liebert UG, Hehme M, Teupser D, Niederwieser D and Al-Ali HK: Highly elevated serum hepcidin in patients with acute myeloid leukemia prior to and after allogeneic hematopoietic cell transplantation: Does this protect from excessive parenchymal iron loading? Adv Hematol 2011: 491058, 2011.

15. Pan X, Lu Y, Cheng X and Wang J: Hepcidin and ferroportin expression in breast cancer tissue and serum and their relationship with anemia. Curr Oncol 23: e24-e26, 2016.

16. Miseta A,Nagy J,Nagy T, Poór VS, FeketeZ and Sipos K: Hepcidin and its potential clinical utility. Cell Biol Int 39: 1191-1202, 2015.

17. Kamai T, Tomosugi N, Abe H, Arai K and Yoshida K: Increased serum hepcidin-25 level and increased tumor expression of hepcidin mRNA are associated with metastasis of renal cell carcinoma. BMC Cancer 9: 270, 2009.

18. Zhang S, Chen Y, Guo W, Yuan L, Zhang D, Xu Y, Nemeth E, Ganz T and Liu S: Disordered hepcidin-ferroportin signaling promotes breast cancer growth. Cell Signal 26: 2539-2550, 2014

19. Ganz T and Nemeth E: Hepcidin and iron homeostasis. Biochim Biophys Acta 1823: 1434-1443, 2012.

20. Schmidt PJ: Regulation of iron metabolism by hepcidin under conditions of inflammation. J Biol Chem 290: 18975-18983, 2015.

21. Kawabata H, Uchiyama T, Sakamoto S, Kanda J, Oishi S, Fujii N, Tomosugi N, Kadowaki N and Takaori-Kondo A: A HAMP promoter bioassay system for identifying chemical compounds that modulate hepcidin expression. Exp Hematol 43: 404-413.e5, 2015.

22. Lee P, Peng H, Gelbart T, Wang L and Beutler E: Regulation of hepcidin transcription by interleukin-1 and interleukin-6. Proc Natl Acad Sci USA 102: 1906-1910, 2005.

23. Wrighting DM and Andrews NC: Interleukin-6 induces hepcidin expression through STAT3. Blood 108: 3204-3209, 2006.

24. Senol K, Ozkan MB, Vural S and Tez M: The role of inflammation in gastric cancer. Adv Exp Med Biol 816: 235-257, 2014.

25. Sobin LH, Gospodarowicz MK and Wittekind C: TNM classification of malignant tumours. Hoboken: Wiley-Blackwell, 2009.

26. Brookes MJ, Boult J, Roberts K, Cooper BT, Hotchin NA, Matthews G, Iqbal T and Tselepis C: A role for iron in Wnt signalling. Oncogene 27: 966-975, 2008.

27. Gozzelino R and Arosio P: Iron homeostasis in health and disease. Int J Mol Sci 17: pii: E130, 2016.
28. Torti SV and Torti FM: Iron and cancer: More ore to be mined. Nat Rev Cancer 13: 342-355, 2013

29. Xue X, Taylor M, Anderson E, Hao C, Qu A, Greenson JK, Zimmermann EM, Gonzalez FJ and Shah YM: Hypoxia-inducible factor- $2 \alpha$ activation promotes colorectal cancer progression by dysregulating iron homeostasis. Cancer Res 72: 2285-2293, 2012

30. Ciniselli CM, De Bortoli M, Taverna E, Varinelli L, Pizzamiglio S, Veneroni S, Bonini C, Orlandi R, Verderio P and Bongarzone I: Plasma hepcidin in early-stage breast cancer patients: No relationship with interleukin-6, erythropoietin and erythroferrone. Expert Rev Proteomics 12: 695-701, 2015.

31. Orlandi R, De Bortoli M, Ciniselli CM, Vaghi E, Caccia D, Garrisi V, Pizzamiglio S, Veneroni S, Bonini C, Agresti R, et al: Hepcidin and ferritin blood level as noninvasive tools for predicting breast cancer. Ann Oncol 25: 352-357, 2014

32. Tesfay L, Clausen KA, Kim JW, Hegde P, Wang X, Miller LD, Deng Z, Blanchette N, Arvedson T, Miranti CK, et al: Hepcidin regulation in prostate and its disruption in prostate cancer. Cancer Res 75: 2254-2263, 2015

33. Marques O, Porto G, Rema A, Rêma A, Faria F, Cruz Paula A, Gomez-Lazaro M, Silva P, Martins da Silva B and Lopes C: Local iron homeostasis in the breast ductal carcinoma microenvironment. BMC Cancer 16: 187, 2016.

34. Nemeth E, Tuttle MS, Powelson J, Vaughn MB, Donovan A, Ward DM, Ganz T and Kaplan J: Hepcidin regulates cellular iron efflux by binding to ferroportin and inducing its internalization. Science 306: 2090-2093, 2004

35. Pinnix ZK, Miller LD, Wang W, D'Agostino R Jr, Kute T, Willingham MC, Hatcher H, Tesfay L, Sui G, Di X, et al: Ferroportin and iron regulation in breast cancer progression and prognosis. Sci Transl Med 2: 43ra56, 2010.

36. Ward DG, Roberts K, Brookes MJ, Joy H, Martin A, Ismail T, Spychal R, Iqbal T and Tselepis C: Increased hepcidin expression in colorectal carcinogenesis. World J Gastroenterol 14: 1339-1345, 2008

37. Ruchala P and Nemeth E: The pathophysiology and pharmacology of hepcidin. Trends Pharmacol Sci 35: 155-161, 2014.

38. Pietrangelo A, Dierssen U, Valli L, Garuti C, Rump A, Corradini E, Ernst M, Klein C and Trautwein C: STAT3 is required for IL-6-gp130-dependent activation of hepcidin in vivo. Gastroenterology 132: 294-300, 2007.

39. Zhao G, Zhu G, Huang Y, Zheng W, Hua J, Yang S, Zhuang J and Ye J: IL-6 mediates the signal pathway of JAK-STAT3-VEGF-C promoting growth, invasion and lymphangiogenesis in gastric cancer. Oncol Rep 35: 1787-1795, 2016.

40. Udali S, Guarini P, Ruzzenente A, Ferrarini A, Guglielmi A, Lotto V, Tononi P, Pattini P, Moruzzi S, Campagnaro T, et al: DNA methylation and gene expression profiles show novel regulatory pathways in hepatocellular carcinoma. Clin Epigenetics 7: 43, 2015.

41. Ock CY, Nam AR, Bang JH, Kim TY, Lee KH, Han SW, Im SA, Kim TY, Bang YJ and Oh DY: Signature of cytokines and angiogenic factors (CAFs) defines a clinically distinct subgroup of gastric cancer. Gastric Cancer 20: 164-174, 2017.

42. Fraenkel PG: Understanding anemia of chronic disease. Hematology Am Soc Hematol Educ Program 2015: 14-18, 2015.

43. Knight K, Wade S and Balducci L: Prevalence and outcomes of anemia in cancer: A systematic review of the literature. Am J Med 116 (Suppl 7A): 11S-26S, 2004.

44. Kim A, Rivera S, Shprung D, Limbrick D, Gabayan V, Nemeth E and Ganz T: Mouse models of anemia of cancer. PLoS One 9: e93283, 2014

45. Park S, Jung CW, Kim K, Kim SJ, Kim WS and Jang JH: Iron deficient erythropoiesis might play key role in development of anemia in cancer patients. Oncotarget 6: 42803-42812, 2015

46. Maccio A, Madeddu C, Gramignano G, Mulas C, Tanca L, Cherchi MC, Floris C, Omoto I, Barracca A and Ganz T: The role of inflammation, iron, and nutritional status in cancer-related anemia: Results of a large, prospective, observational study. Haematologica 100: 124-132, 2015.

This work is licensed under a Creative Commons Attribution-NonCommercial-NoDerivatives 4.0 International (CC BY-NC-ND 4.0) License. 